\section{Isolation of Tetramethylpyrazine from Culture of Bacillus natto, and Biosyn- thetic Pathways of Tetramethylpyrazine}

As has been shown in the previous communication ${ }^{1}$, we have obtained tetramethylpyrazine from cultures of a strain of Bacillus subtilis and it had the characteristic smell of fermented soybean. The fermented soybean is the favourite food as 'natto' among Japanese people. The isolation of tetramethylpyrazine from 'natto' has been described ${ }^{2}$. Since Bacillus natto is a bacterium which ferments soybean to produce 'natto', we made an attempt to isolate tetramethylpyrazine from cultures of this bacterium.

After cultivating the bacterium for a few weeks in a medium containing glucose, peptone and meat extract, the whole cultures were subjected to flash evaporation. Water was evaporated out and the distillate extracted with ether. By addition of suitable amounts of picric acid to the ether extract a picrate was obtained. It was confirmed that the picrate is identical with an authentic sample of tetramethylpyrazine picrate by mixed melting points and comparisons of their infra-red spectra. This proved that Bacillus natto produces tetramethylpyrazino as does Bacillus subtilis.

The biosynthetic pathways of tetramethylpyrazine are of interest, since it was the second pyrazine to be discovered as a natural product and we supposed that the biosynthotic pathways differ somewhat from the pathways of the first-discovered pyrazine, aspergillic acid.

Baxter and Spring ${ }^{3}$ have reported that aspergillic acid is biosynthesized from isoleucine via the diketopiperazino. But it is difficult to propose a plausible mechanism for the pathways from amino-acid to tetramethylpyrazine, since the hydroxy group should remain on the pyrazine nucleus instead of the methyl if it was biosynthesized from amino-acids.

In our previous communication ${ }^{1}$, it was reported that tetramethylpyrazine was obtained after cultivation of the bacterium in media with asparagine as a source of nitrogen. The same compound was obtained when the bacillus cultivated in medias with arginine, aspartic acid, alanine, glycine, glutamic acid and even ammonium chloride, rospectively, as nitrogen source.

The results might suggest that those amino-acids would be utilized as only amine parts of pyrazine and carbon parts might be converted from another source, possibly sugars.

In some micro-organisms acetoine is synthesized from sugars via pyruvic acid, and it was found by means of the Voges-Proskauer test that both Bacillus subtilis and $B$. natto also produced acetoine in detect. able amounts.

It is thought from the chemical point of view that tetramethylpyrazine might be biosynthesized from acetoine and the amino part of amino-acids. A plausible scheme for the biosynthetic pathways is given thus:<smiles>CCC(=N)C(C)O</smiles>

Further proof for the mechanism is needed and we are working on this.

TAKuo Kosuge

TaHeI Adachi

Hiroko KaMrYA

Shizuoka College of Pharmacy,

Shizuoka,

Japan.

${ }^{1}$ Kosuge, T., and Kamiya, H., Nature, 193, 776 (1962).

${ }^{2}$ Kosuge, T., Kamiya, H., and Adachi, T., J. Pharm. Soc. Japan, 82 190 (1962).

${ }^{3}$ Baxter, R. A., and Spring, F. S., J. Chem. Soc., 1179 (1947),

\section{PHYSIOLOGY}

\section{Percutaneous Absorption of Nicotinic Acid and Ethyl Nicotinate in Human Skin}

This communication concerns the vast difference in percutaneous absorption through human skin of two related substances, nicotinic acid and ethyl nicotinate.

Human skin removed surgically from breasts, legs, and arms was used within $4 \mathrm{~h}$ of removal. Great care was exercised in handling the specimen, and the subcutaneous fat was carefully cut away with scissors. The skin was draped over a funnel and secured with elastic bands, the corium being directed to the inside of the funnel and the epidermis to the outside. The substance to be measured was applied to the epidermis, and the funnel with the epidermis downward was placed on wooden strips in a Petri dish. When using ethyl nicotinate, because of its volatility, aluminium foil was used to cover the epidermis and the cut sides of the skin were occluded with adhesive tape. Saline was injected into the funnel so that it bathed the corium and the narrow opening of the funnel was covered. Aliquots of saline were withdrawn periodically for quantitative determinations.

Both nicotinic acid and ethyl nicotinate were labelled with carbon-14 in the carboxyl groups. The specific activity of the nicotinic acid samples was $\mathbf{9 \cdot 2}$ $\mathrm{mc} . / \mathrm{mmole}$ and $6.8 \mathrm{mc} . / \mathrm{mmole}$; the sample of ethyl nicotinate was $6.77 \mathrm{mc} . / \mathrm{mmole}$. $0.1 \mathrm{c.c}$. nicotinic acid and 0.01 c.c. ethyl nicotinate were pipetted on to the epidermis. With nicotinic acid the excess fluid was dried off the skin surface before inverting the funnel into a Petri dish; with ethyl nicotinate the skin was covered with aluminium foil before inversion. At regular intervals an aliquot was removed from the saline bathing the corium and placed in a planchet for counting with a Nuclear-Chicago gas-flow thinwindow Geiger counter. Ethyl nicotinate was con. verted to ethyl nicotinate hydrochloride by previously. adding two drops of $1 \mathrm{~N}$ hydrochloric acid to the planchet. The percentage of substance recovered was determined by measuring the counts obtained in the saline bathing the corium and comparing this with the number of counts applied to the epidermal surface. In order to determine the rate of penetration of skin without epidermis, the epidermis was removed by heating the skin to $63^{\circ} \mathrm{C}$ on a hot plate for $2 \mathrm{~min}$. after which the epidermis peels away from the corium with ease. The radioactive compounds were then applied directly to the surface of the dermis and in the same way aliquots were taken at regular intervals. Percentage penetration through skin without epidermis was thus calculated for both compounds.

Twenty-four pieces of skin from 12 different specimens were used for nicotinic acid, and 14 pieces from 